\title{
UAF: a generic OPC Unified Architecture Framework
}

\author{
Wim Pessemier $^{a}$, Geert Deconinck $^{b}$, Gert Raskin $^{a}$, Philippe Saey ${ }^{b, c}$ and Hans Van Winckel ${ }^{a}$ \\ ${ }^{a}$ Institute of Astronomy, KULeuven, Leuven, Belgium; \\ ${ }^{b}$ ESAT - dept. of Electrical Engineering, KULeuven, Leuven, Belgium; \\ ${ }^{c}$ E \& A - Energy \& Automation, KAHO St.-Lieven, Gent, Belgium
}

\begin{abstract}
As an emerging Service Oriented Architecture (SOA) specifically designed for industrial automation and process control, the OPC Unified Architecture specification should be regarded as an attractive candidate for controlling scientific instrumentation. Even though an industry-backed standard such as OPC UA can offer substantial added value to these projects, its inherent complexity poses an important obstacle for adopting the technology. Building OPC UA applications requires considerable effort, even when taking advantage of a COTS Software Development Kit (SDK). The OPC Unified Architecture Framework (UAF) attempts to reduce this burden by introducing an abstraction layer between the SDK and the application code in order to achieve a better separation of the technical and the functional concerns. True to its industrial origin, the primary requirement of the framework is to maintain interoperability by staying close to the standard specifications, and by expecting the minimum compliance from other OPC UA servers and clients. UAF can therefore be regarded as a software framework to quickly and comfortably develop and deploy OPC UA-based applications, while remaining compatible to third party OPC UA-compliant toolkits, servers (such as PLCs) and clients (such as SCADA software). In the first phase, as covered by this paper, only the client-side of UAF has been tackled in order to transparently handle discovery, session management, subscriptions, monitored items etc. We describe the design principles and internal architecture of our open-source software project, the first results of the framework running at the Mercator Telescope, and we give a preview of the planned server-side implementation.
\end{abstract}

Keywords: OPC UA, software framework, PLC

\section{MOTIVATION}

As the original OPC (Object Linking and Embedding for Process Control) communication technology suffered from some serious limitations (mostly inherited from its underlying, Microsoft Windows dependent DCOM technology), its usage in telescope control systems remains very limited. The follow-up Unified Architecture standard (OPC UA) on the other hand quite radically deals with many of the issues related to its predecessor and furthermore introduces a substantial amount of new features. This justifies a fresh look at the self-declared "next generation OPC standard that provides a cohesive, secure and reliable cross platform framework for access to real time and historical data and events". ${ }^{1}$ While we indeed found OPC UA to be sufficiently capable and technologically ready to serve as the main communication technology to reliably (but non-deterministically) integrate the components of an observatory control system, ${ }^{2}$ it remains to be seen whether - and at which pace - industry will be picking up on this recent technology.

Having this said, a number of leading manufacturers of industrial automation equipment and software have already added OPC UA interfaces to their portfolio, which has increased the surviving chances of the technology significantly. Considering the many advantages the new technology has, organizations that depend on products of these "early adopting" manufacturers may well choose to migrate from their existing OPC (or proprietary protocol based) solutions towards the new Unified Architecture standard. ${ }^{3}$ At the Mercator Telescope, a $1.2 \mathrm{~m}$ optical telescope based at the Roque de los Muchachos Observatory at the Canary Islands, we found OPC UA to be the most logical choice to become the main communication technology of our observatory control system since it is well supported by the Beckhoff PLCs which have been selected as the main platform to control the telescope, the instruments and most other observatory-related tasks. In addition, we are currently evaluating the

wim.pessemier@ster.kuleuven.be 
Siemens WinCC SCADA software packages (versions 7, 11 and Open Architecture) to supervise our Telescope Control System (TCS), and these also support OPC UA communication.

Another reason to include OPC UA technology in our Mercator Observatory Control System (MOCS) is to migrate from the currently running "custom" solution towards a more standardized "industrial" solution in the future. The current MOCS is based on a project-specific framework built around a bare-bone "IT-centric" middleware, in contrast to OPC UA which defines a rich information model (including IEC standardized models for alarms, sensor values, devices, state machines, etc.) that can be extended cleanly by using namespaces, and that is already re-used in many other projects in industry.

As a final argument, by using OPC UA, we also expect to increase the dependability of our systems. OPC UA has built-in support for secure communication and in many ways favors dependability over achieving the maximum attainable performance (as can be seen for instance by the "heartbeat" messages that are sent between subscribers and publishers in both directions). In the same context, we consider the availability of COTS OPC UA-compliant products as a significant advantage. At the Mercator telescope we have already installed two PLCs running OPC UA and we may install a commercial OPC UA-capable SCADA software package soon. These two COTS solutions alone enable us to build advanced OPC UA servers and clients respectively, with little or no error-prone programming efforts involved. Likewise, the UAF is built on top of a mature and stable commercial OPC UA Software Development Kit (SDK).

While such an OPC UA SDK provides all necessary "building blocks" to develop dependable OPC UA applications, it is up to the user of the SDK to assemble these blocks and add business logic in order to build a functional application. This implies that a lot of technical concerns still have to be handled by the application developer. To allow the developer to concentrate on the business logic of the application, we chose to design the Unified Architecture Framework as a reusable abstraction layer between the SDK and the functional application code. The framework should exhibit a stable and well-defined behavior at the cost of loss of flexibility.

The framework is developed as an open-source project, although one should note that a commercial OPC UA Software Development Kit must be obtained in order to be able to compile the source code (see section 3.2). As the framework can be compiled against an online available (albeit restricted in time) demo version of this SDK, it is possible to test the framework for free when agreeing with the license of the SDK supplier. The source code of the UAF can be found on the following website: http://www.ster.kuleuven.be/uaf.

\section{FRAMEWORK PHILOSOPHY}

The UAF is called a framework (instead of a toolkit or library) since it complies to the principle of inversion of control, ${ }^{4}$ and since it defines a default behavior that is easy to extend but hard to modify. A problem often seen with observatory frameworks (and software frameworks in general) is that they tend to turn into "exclusive" solutions for a particular project as they evolve, making them difficult to maintain and to support on different platforms. The UAF attempts to avoid this pitfall by not trying to be the software framework for a given project, but rather $a$ software framework to conveniently develop interoperable control system solutions on a well-defined set of platforms. This approach is a way to prevent "frameworkitis", as mentioned in an interview series ${ }^{5}$ with Erich Gamma, one of the authors of the Gang of Four book. ${ }^{6}$ According to Gamma,

frameworkitis is the disease that a framework wants to do too much for you or it does it in a way that you don't want but you can't change it. (...) Also, if we do frameworks, we try to make them small frameworks. We prefer many small frameworks over one heavyweight framework.

The UAF is in that sense an example of a framework that prevents application developers from catching "frameworkitis" since it does not pretend to be a "one size fits all" solution. UAF is only one way to build an OPC UA compliant application. Developers wishing more freedom can build their OPC UA applications directly on top of one of the available OPC UA Software Developers Kits, PLC programmers may expose their software directly via a commercial OPC UA server, and User Interface (UI) designers may even develop OPC UA client applications using a commercial SCADA software package. 
The key to maintain interoperability is to rigorously stick to OPC UA's standard data encoding and transport specifications on the one hand, and OPC UA's standard information model on the other hand. The former is guaranteed by building OPC UA on top of a commercial SDK, while the latter requires the UAF developer(s) to be cautious when inserting new features. The UAF may handle a lot of the technical concerns transparently to the application developer (such as automatically creating sessions and subscriptions when needed), but it should not expect more from its environment than what is strictly defined by the OPC UA specifications. On a practical level, the UAF should also be designed with the present state of commercially available OPC UA products in mind. It may be of little use to create UAF applications supporting more advanced features such as state machine models and dynamic address spaces, if the third-party products that need to interact with them do not exist (yet).

\section{TECHNICAL DESCRIPTION (CLIENT SIDE)}

In this section we will give an overview of the implementation details of the UAF. Section 3.7 about the top-level API contains an example which might be sufficient to allow developers to start experimenting with the UAF, although reading the other sections will certainly help to get a better understanding of how the framework works internally. Compound words of the OPC UA or UAF terminology (such as UnresolvedExpandedNodeId) will be written in italics to enhance clarity.

\subsection{Overview}

Since both the client and server side deal with a common terminology (such as sessions, subscriptions, events, discovery, ...), the following namespaces have been defined to prevent name collisions:

- uafc: client side of the UAF;

- uafs: server side of the UAF;

- uafcs: client and server functionality (such as configurable aggregating servers);

- uaf: common utility functionality used by the other namespaces.

At the time of writing this paper (the beginning of June 2012) only the uafc and uaf namespaces contain functional code. The uafs and uafcs namespaces on the other hand will be developed in the coming months.

Often recurring throughout the description below and throughout the UAF source code is the concept of NodeIds. As will be explained more in detail in section 3.4, the address space of an OPC UA server consists of "nodes" (information entities representing an object, a variable, a method, etc.) interconnected by "references" (relationships representing composition, inheritance, type definition, etc.). Nodes contain attributes such as an identifier (the NodeId) and a name that can be used for browsing through the address space (the BrowseName), both of them defined according to a well-defined namespace. For efficiency reasons however, OPC UA servers do not expose the full URI of this namespace for each node, but instead they map the namespace URI to a simple integer, the namespace ID. Clients are able to resolve nodes because they can read the mapping between the URIs and IDs, which is exposed by every server in a so-called NamespaceArray. Similarly, if a node is referenced to a particular server, the OPC UA specifications define an ExpandedNodeId which only contains the numerical ID of this server. Clients can read the ServerArray to find out which server ID belongs to which server URI.

Because node resolution is such an important part of OPC UA client-server communication, we have defined a clear terminology for the UAF. By explicitly distinguishing between the resolved or unresolved state of a node, and between the local or system-wide referencing, four new classes have been added to the framework (see Fig. 1). This allows application developers to simply provide UnresolvedNodeIds (comparable to the "definitions" of these NodeIds) to the framework. The UAF will then internally convert them into the resolved equivalents whenever necessary, cache them for resource optimization, renew them in case a server fails and a redundant server takes over, etc. 


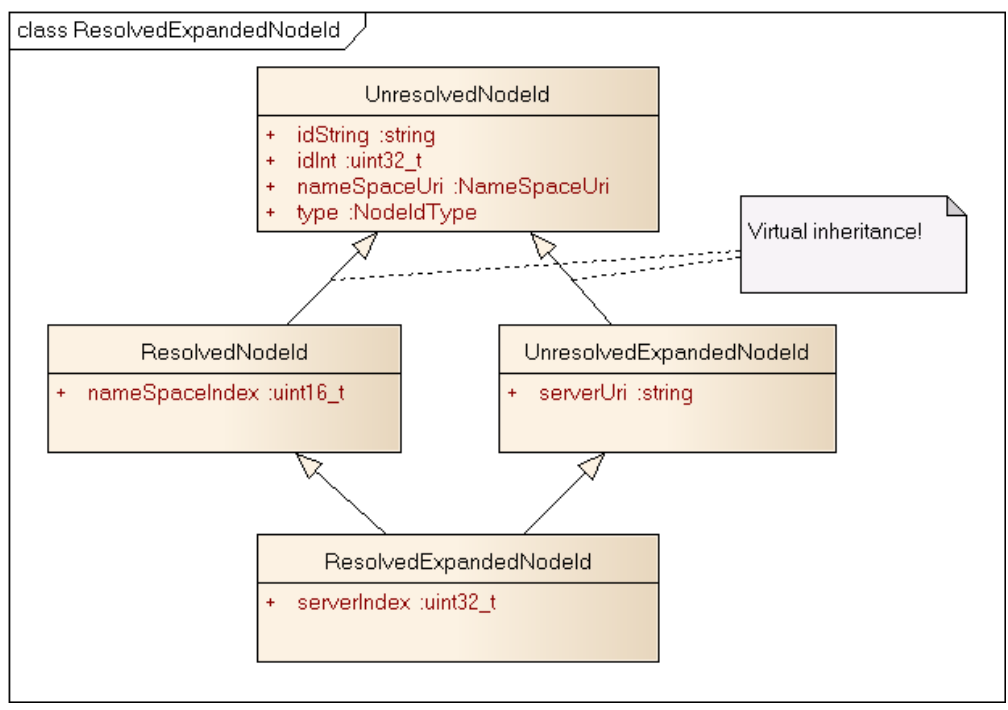

Figure 1. UML model of the ResolvedExpandedNodeId inheritance tree.

\subsection{Dependencies}

Similar to the underlying C++ SDK, UAF has been developed with portability and maintainability in mind, resulting in a minimal set of dependencies. This means that for $\mathrm{C}++$ application development, no additional dependencies need to be installed apart from the obvious SDK and the third-party software packages required by the SDK. If SWIG and the Python development libraries are available on the target system, the UAF can also provide a Python interface. A summary of the current dependencies is listed below:

- OPC UA SDK (32-bit, C++, Linux) by Unified Automation: this commercial OPC UA Software Development Kit is UAF's foremost dependency. The SDK provides all necessary "building blocks" to be wrapped, extended and assembled by the UAF. A demo version can be downloaded freely, and can be used for evaluation purposes since it allows unrestricted usage for 1 hour (after which the software must be restarted). For each individual developer, a binary or a source code license of the SDK can be purchased. The latter is the more expensive option but offers the freedom to compile the SDK on a wide range of platforms, and may better protect software investments for the future. The Unified Automation SDK depends on the following third-party software projects:

- LibXML2: An optional dependency of the Unified Automation SDK, but mandatory for UAF because the latter uses XML to read all configuration files.

- OpenSSL: Again an optional requirement of the OPC UA SDK, since it is only required if security features need to be available. Because UAF has been developed for use cases involving secure communication, OpenSSL is currently a mandatory dependency.

- CMake: The SDK uses CMake as its build system, and UAF has followed this decision. CMake generates native make-files from platform and compiler independent configuration files and thus offers a convenient way to compile the SDK and UAF on multiple platforms. While the UAF has only been tested on Linux, we expect few problems to adapt the software to other platforms if such a use case appears in the future.

- SWIG: The Simplified Wrapper and Interface Generator is only required if a Python API must be provided by the UAF.

- Python development libraries: Needed by SWIG to build the Python wrappers. 


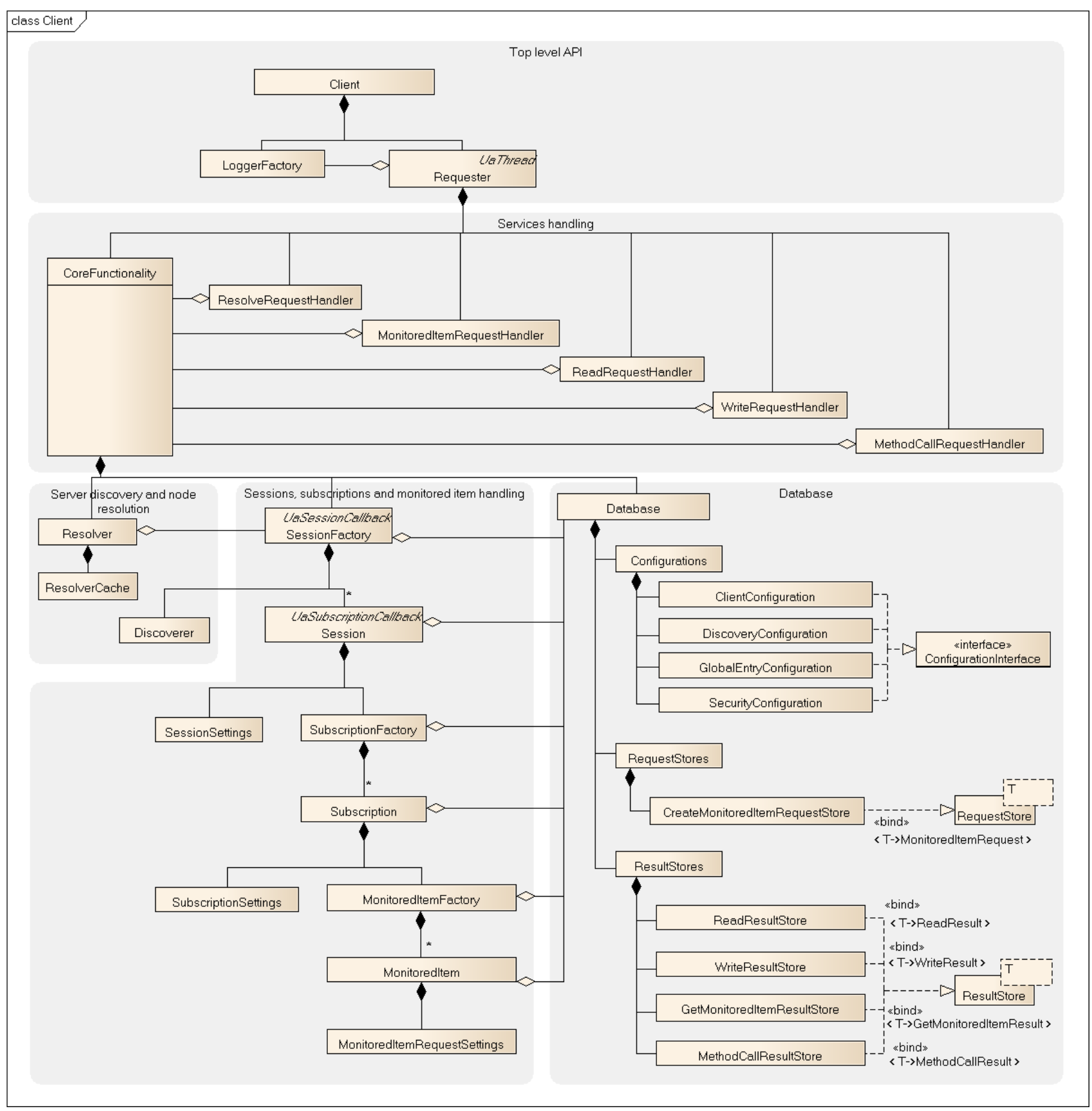

Figure 2. UML model of the Unified Architecture Framework.

\subsection{Session, subscription and monitored item handling}

As mentioned before, OPC UA SDKs offer full flexibility to the end-user but require a substantial amount of technical concerns to be handled. The gray area in Fig. 2 carrying the same title as this section shows how the UAF attempts to relieve the developer from the assembly of some of the most important "building blocks" of the SDK. These building blocks include sessions (communication context), subscriptions (asynchronous communication channels that use these sessions), and monitored items (notifications of data changes and events that use these subscriptions). UAF effectively wraps session and subscription instances, stores them in memory, 
implements their corresponding callback interfaces, and simplifies their management by providing an interface which hides many purely technical concerns. As can be derived from Fig. 2, sessions, subscriptions and monitored items are handled in a very similar way:

- Software factories offer a convenient way to create, delete and look up instances, and to perform operations (such as "disconnect") on all created instances in a single optimized call. The software factories also try to optimize resource usage internally by only creating an instance if a similar one does not exist already. For instance, when a subscription should be created to accommodate a new monitored item, the factory will only create one if no other subscription with the exact same settings already exists for the particular session.

- The UAF automatically assigns an ID to all sessions, subscriptions and monitored items. This ID is considered a technical concern and is therefore only used internally by the SDK and the UAF (e.g. to associate callbacks with sessions), so end-users of the UAF do not have to deal with them.

- The UAF stores the settings of each session/subscription/monitored item to allow the software factories to optimize resource usage.

- Sessions, subscriptions and monitored items also expose their associated services to the rest of the framework.

\subsection{Server discovery and node resolution}

One of the most interesting features of OPC UA is the support for complex information models, composed of information entities ("nodes") interconnected by relationships ("references"). These relationships can have any semantics - not just aggregation and inheritance relationships as is most often the case with other middleware technologies. References are most commonly connecting two nodes on the same server, but they may also define relationships between nodes exposed by separate servers (in order to build a "system-wide" information model for instance).

Clearly, in a potentially complex network as described above, there can be numerous ways (or BrowsePaths according to OPC UA terminology) to locate a particular information entity. Instead of a simple tree-like information model, OPC UA servers may expose a mesh topology of nodes related to other nodes (existent or non-existent, on the same or another server) by references of any semantics.

While it remains an interesting challenge to take advantage of the OPC UA support for references exposing semantics other than aggregation and inheritance, the UAF currently supports several more common ways to locate a particular node on a server discovered by the OPC UA discovery mechanism. Our framework defines and supports the following node resolution use cases:

- by UnresolvedExpandedNodeId: server URI and namespace URI of the NodeId are known, but the server URL and namespace ID are not. In other words, the "definition" of the server and NodeId are both given, but the exact server URL still needs to be discovered, and the exact NodeId still needs to be determined based on the server's NamespaceArray.

- by ResolvedExpandedNodeId: similar to the previous use case, but the NodeId is now fully known in advance. Since the namespace URI must not be translated first into a namespace ID, a client can resolve this node more quickly. However, one must be careful when storing ResolvedExpandedNodeIds persistently, since the namespace ID is bound to a particular configuration of a particular server and thus may change in the future.

- by GlobalBrowsePath: in this case the client follows a "path" of BrowseNames of hierarchically connected nodes, starting from a known node (the so called GlobalEntryPoint). The GlobalBrowsePath and GlobalEntryPoint terminology is not part of the OPC UA specifications but it is introduced by the UAF to specify a BrowsePath spanning over multiple servers and relative to a single well-defined node (typically exposed by a redundant server). Each system or subsystem consisting of multiple servers may define such a GlobalEntryPoint in order to be able to reference the (root) nodes of the servers relative to a single well-known node, the GlobalEntryPoint. Clients based on the UAF may specify this node by configuration. 
- by BrowsePath: similar to the previous use case, only the starting point of the BrowsePath is not known to the UAF (via configuration) and thus must be provided by the application developer.

- by ExpandedBrowsePath: again a term defined by UAF, this browse path is (analogous to the OPC UA definition of an ExpandedNodeId) associated with a given server URI. The starting point of this BrowsePath is the root node of the server, as defined by the OPC UA namespace.

Discovery of servers in OPC UA is accomplished by having the client invoke the FindServers service on a known discovery server. The discovery server will then return the application descriptions of the known servers (or other discovery servers in case a hierarchy of discovery servers exists in the network). The client can subsequently request the so-called endpoint descriptions of each server individually, and create a session to one of these endpoints afterwards.

In none of the above use cases, a server URL must be specified since at least one URL (either the URL of a discovery server or the URL of the server hosting the node) must be provided to the client by configuration. Server discovery and node resolution is thus handled transparently to the client. On a side note, the new terminology introduced by the UAF does not lead to "frameworkitis" as mentioned before, since the UAF only introduces new names for existing concepts in order to simplify its API.

\subsection{Database}

As can be seen from Fig. 2, a uafc: :Database instance is part of the core functionality of UAF clients. This database can be accessed by nearly the whole UAF client side since a pointer to the database instance is passed to most parts of the framework. Various kinds of information are stored inside the database:

- Configurations: container objects storing configuration parameters such as:

- the URLs of the discovery servers that should be known to the client;

- security related settings such as the location of the certificate store, the client certificate details, the certificate revocation list, etc.;

- the GlobalEntryPoint node details;

- various other client related settings such as the interval between subsequent server discovery attempts.

Each configuration object can be populated by loading a specific XML file, or by setting the parameters directly via method calls. In a secure environment, one can choose to make the XML files for discovery and global entry available to all clients via a shared network disk, since these files can describe system wide information.

- Request stores: container objects storing requests (such as CreateMonitoredItemRequests) so that the state of the client can transparently be (re)created in case of failures. Request stores mark requests either as created on the client side, or not. This means that a CreateMonitoredItemRequest marked as created on the client will have a corresponding session, subscription and monitored item assigned. If the request is not marked as created on the client on the other hand, the UAF will periodically attempt to retrieve the necessary information (by server discovery and node resolution) and eventually create a suitable session, subscription and monitored item. Applications using UAF can thus for example issue a request to monitor some variables, even if this server is not available yet. Likewise, when the lifetime of the associated subscription or session receives a timeout, the monitored item instance is automatically deleted from the client side, and its associated request is scheduled for re-creation. Since the Node resolution of the monitored item is performed again, the monitored item may even be created on another (redundant) server - fully transparent to the client application.

- Result stores: container objects storing the results from asynchronous requests. Asynchronous requests may be monitored items (data and events), method calls, or asynchronous read or write requests. 


\subsection{Services handling}

The services supported by UAF are handled in a generic way using so-called "request handlers". One request handler is created for each set of related services. The MonitoredItemRequestHandler for instance accepts CreateMonitoredItemRequests and DeleteMonitoredItemRequests, and returns CreateMonitoredItemResults and DeleteMonitoredItemResults respectively. As can be seen in Fig. 2, request handlers currently exist for reading data, writing data, monitoring data, monitoring events, calling methods, and for explicitly resolving nodes. Others may be added in the future since this part of the framework is very easy to extend.

\subsection{Top level API}

The client functionality is exposed to the higher level application by three levels of abstraction:

1. Request handlers. As already mentioned in section 3.6, the UAF defines request handlers which expose a consistent API via the exchange of requests and corresponding results. Even though these request handlers require more code to be written by the application developer, they offer most flexibility and they can be matched with code generators more easily.

2. The Requester. This object exposes a simplified API (including methods such as read() and write()) and translates the incoming and outgoing data to the corresponding requests and results of the underlying request handlers.

3. The Client. The top level API is very similar to the Requester API but hides some of the UAF implementation details. For instance, the Client interprets the status codes returned by the Requester and uses exception handling instead to handle errors.

The UAF also contains SWIG interface files to wrap the top level C++ API with a Python API. A usage example of this Python interface is shown in Fig. 3. This example is not randomly chosen but in fact corresponds to the recently commissioned PLC-based control system of the tertiary mirror of the Mercator Telescope, as mentioned in the next section and as described in a dedicated paper. ${ }^{7}$

\section{USE CASES}

At the time of writing, June 2012, the UAF is used at the Mercator Telescope for two systems:

- Supervision of the tertiary mirror (M3) control. The motion control of the new tertiary mirror support for the Mercator telescope is implemented on a Beckhoff PLC and exposed by a Beckhoff OPC UA server running on the same platform. A simple graphical client written in Python/Qt uses the UAF to connect to this server, to display the actual values and configuration values, and to trigger operations (such as "go to Nasmyth A"). This user interface has been written as a quick temporary solution since it may be replaced by a commercial SCADA application (such as WinCC OA by Siemens) in the near future.

- Telemetry acquisition of the MAIA instrument. Very similar to the M3 control, the instrument control of the three-channel camera MAIA is fully implemented on a Beckhoff embedded PC hosting the PLC run-time, a Human Machine Interface (HMI) and an OPC UA server. A non-graphical Python client based on UAF connects to this PLC via OPC UA, monitors some data variables, and stores them periodically in the central database of the Mercator observatory.

A paper describing these control systems in much more detail has been published. ${ }^{7}$ The combination of PLC-based control and UAF-based supervision allowed us to create very stable systems with little effort, since most of the technical concerns of the server side are handled by the PLC run-time environment, and most of the technical concerns of the client side are handled by the framework. A downside of this approach is that the particular (non object-oriented) programming model of PLCs is reflected in the address space exposed by the OPC UA server of the PLC. This may hamper the integration of PLC systems in the overall system since high level systems are often modeled according to object-oriented design principles. To deal with this problem, we have developed a model-driven approach that allows us to expose IEC61131-3 software via OPC UA in a very structured, "object-oriented-like" way. The full description of this methodology can be found as an appendix to the previously mentioned paper. 
Import the framework and define the namespace URI and server URI:

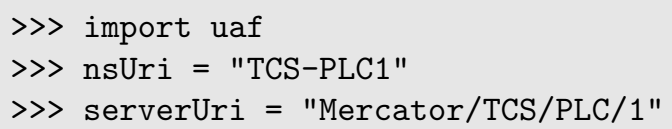

Define nodes by UnresolvedExpandedNodeId, or by ResolvedExpandedNodeId, or by BrowsePath, or by ExpandedBrowsePath, or by any other supported method:

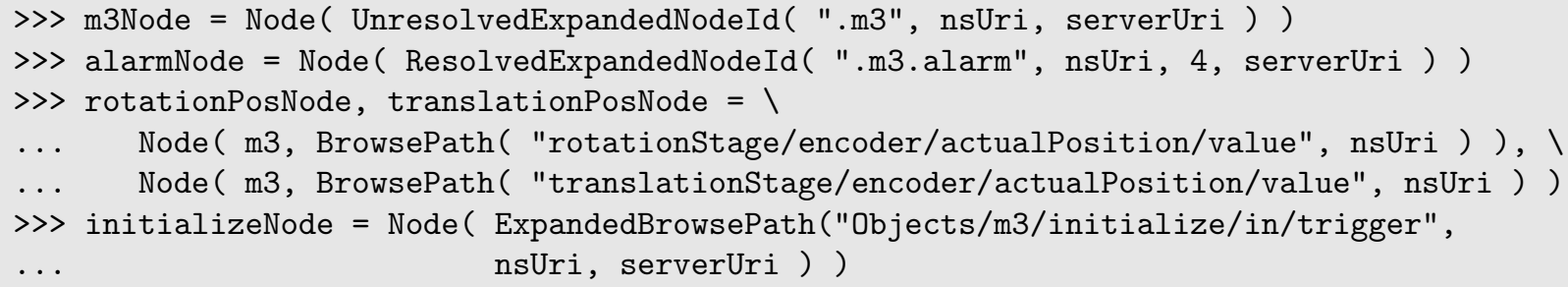

Implement the client:

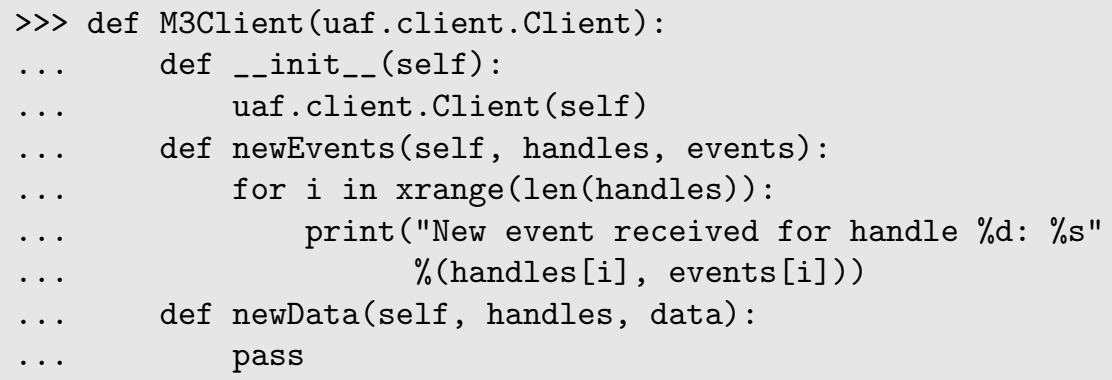

Client instances will print a message for each incoming event. The overridden newData() method is not implemented since (in this example) we're not interested in when the data changes. Instead, we can just use the get() functionality provided by the UAF client to fetch the latest received data.

Test the client:

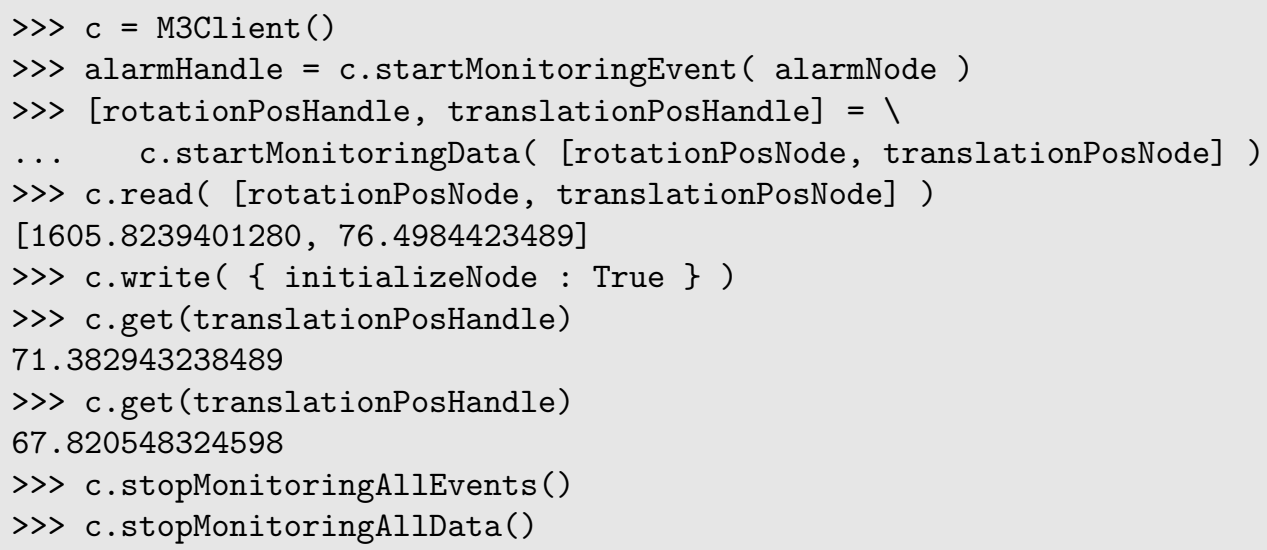

Figure 3. Usage example of the Python API of the Unified Architecture Framework. 


\section{FUTURE DEVELOPMENTS}

In the second half of 2012 we envision to continue the development of the UAF and start implementing also the server side. The design rules and framework philosophy remain the same, meaning that we aim to create an abstraction layer that will help us to create OPC UA-compliant server applications with minimal effort. When both client and server implementations are finished, we'd like to shift our attention to so-called "aggregating servers". These servers are in fact clients to other servers, and act as a façade ${ }^{8}$ for them. Aggregating servers can take care of fine-grained security measures (such as role-based access control) and can even influence the reproduced address space (by adding OPC UA views for instance) of the underlying servers on-the-fly.

\section{CONCLUSIONS}

As a non-project specific software package created to assist the development of OPC UA-compliant applications, the Unified Architecture Framework currently lives up to its own generic name. The UAF is a framework in the true sense of the work since it offers callback mechanisms and since it defines a default behavior that is constraining the freedom of end-users, but at the same time relieving them from most of the technical burden associated with OPC UA application development. The UAF is specifically designed with interoperability to commercially available OPC UA compliant products in mind. At the Mercator Telescope, we plan to control and supervise most of our instrumentation via OPC UA-enabled PLCs and OPC UA-enabled commercial SCADA software. The new framework on the other hand will be used only for the "niche" applications for which a Linux/PC platform and a popular programming language such as $\mathrm{C}++$ or Python is the most convenient solution. The first client applications based on the UAF are already running at the Mercator Telescope site, and are demonstrating the effectiveness of an instrumentation control system based on PLCs and an advanced industrial communication technology such as OPC UA. This convinces us to continue our development efforts in the future, so we aim to start the development of the server-side of the framework during the second half of 2012 .

\section{REFERENCES}

[1] OPC Foundation, "OPC UA," (2012). http://www.opcfoundation.org/Default.aspx/01_about/UA.asp.

[2] Pessemier, W., Raskin, G., Van Winckel, H., Deconinck, G., and Saey, P., "Suitability Assessment of OPC UA as the Backbone of Ground-based Observatory Control Systems," International Conference on Accelerator and Large Experimental Physics Control Systems (ICALEPCS) THAAUST02 (2011).

[3] Farnham, B. and Barillère, R., "Migration from OPC-DA to OPC-UA," International Conference on Accelerator and Large Experimental Physics Control Systems (ICALEPCS) MOPMS025 (2011).

[4] Martin Fowler, "InversionOfControl (blog)," (2005). http://martinfowler.com/bliki/InversionOfControl.html.

[5] Venners, B. and Gamma, E., "Erich Gamma on Flexibility and Reuse - A Conversation with Erich Gamma, Part II," (2005). http://www.artima.com/lejava/articles/reuse.html.

[6] Gamma, E., Helm, R., Johnson, R., and Vlissides, J., [Design Patterns: Elements of Reusable Object-Oriented Software], Addison-Wesley (1994).

[7] Pessemier, W., Deconinck, G., Raskin, G., Saey, P., and Van Winckel, H., "Design and First Commissioning Results of PLC-based Control Systems at the Mercator Telescope," in [Software and Cyberinfrastructure for Astronomy II: Technology Advancements], Proc. SPIE 8451 (2012).

[8] Hohpe, G. and Woolf, B., [Enterprise Integration Patterns - Designing, Building and Deploying Messaging Solutions], Addison-Wesley (2011). 